\title{
Thrombocytopenic Purpura after the Administration of an Influenza Vaccine in a Patient with Autoimmune Liver Disease
}

\author{
Satoshi Mamori Katsuyuki Amano Hiroyuki Kijima Ichiro Takagi Hisao Tajiri \\ Division of Gastroenterology and Hepatology, Department of Internal Medicine, The Jikei University School of \\ Medicine, Tokyo, Japan
}

\section{Dear Sir,}

Influenza, a contagious viral disease, can usually be prevented by receiving an influenza vaccine. Almost 15 million doses of influenza vaccine were produced in Japan for the 2003-2004 influenza season. Although serious side effects from the influenza vaccine are extremely rare, the influenza vaccine, as all vaccines, may sometimes induce or trigger autoimmune reactions in susceptible individuals (e.g., the development of Guillain-Barré syndrome) [1]. This report describes a patient with autoimmune liver disease (AILD) who developed idiopathic thrombocytopenic purpura (ITP) after receiving an influenza vaccine.

In August 2005, a 75-year-old female living in Tokyo was referred to the Jikei Daisan Hospital because of abnormal liver function test results. She had no history of daily alcohol consumption or obesity. The physical examination was unremarkable. Her laboratory data showed: aspartate aminotransferase (AST), $70 \mathrm{IU} / \mathrm{l}$; alanine aminotransferase (ALT), 40 IU/l; lactate dehydrogenase (LDH), 222 IU/l; alkaline phosphatase (ALP), $266 \mathrm{IU} / \mathrm{l} ; \gamma$-glutamyl triphosphate $(\gamma$-GTP), $200 \mathrm{IU} / \mathrm{l}$, and total bilirubin (T-Bil), $1.0 \mathrm{mg} / \mathrm{dl}$ (normal ranges: AST, 10-33 IU/1; ALT, 6-35 IU/1; LDH, 130-235 IU/l; ALP, 96-300 IU/1; $\gamma$-GTP, 9-27 IU/l; T-Bil, 0.2-1.2 mg/dl). The peripheral blood cell counts were as follows:
RBC, $6,600 / \mu \mathrm{l} ; \mathrm{WBC}, 4.15 \times 10^{6} / \mathrm{ml} ; \mathrm{Hb}$, $13.2 \mathrm{~g} / \mathrm{dl} ; \mathrm{Ht}, 39.5 \%$; Plt, $164 \times 10^{3} / \mathrm{ml}$ (normal ranges: $\mathrm{RBC}, 4,500-8,500 / \mu \mathrm{l}$; WBC, $3.80-4.80 \times 10^{6} / \mathrm{ml}$; Hb, 11.5-14.5 g/dl; Ht, 35.0-42.0\%; Plt, 150-350 × 103/ $\mathrm{ml})$. The serological findings showed: antinuclear antibody (ANA), titer 1/320 (normal value, $<1 / 20$ ); antimitochondrial antibody (AMA), titer $1 / 160$ (normal value, <1/20); IgG, 3,180 mg/dl; IgM, 1,231 mg/dl (normal ranges: IgG, $870-1,700 \mathrm{mg} / \mathrm{dl}$; IgM, 35-220 mg/dl). Moreover, anti-hepatitis B surface antibody and anti-hepatitis $\mathrm{C}$ antibody were negative. The ultrasound examination findings did not show any evidence of a specific disease causing the abnormal liver function, such as a liver tumor. She was diagnosed as having AILD and was treated with ursodeoxycholic acid (UDCA; $10 \mathrm{mg} / \mathrm{kg}$, daily). Three months later, her liver function returned to almost normal levels (AST, 38 IU/l; ALT, $20 \mathrm{IU} / \mathrm{l})$.

On January 23, 2006, she presented at an outpatient clinic with epistaxis and petechiae on her lower extremities. No evidence of lymphadenopathy or hepatosplenomegaly was noted. She had received an influenza vaccine (Influenza HA Vaccine 'Kitaken') 1 week before the onset of her symptoms. Upon admission, her platelet count was $5 \times 10^{3} / \mathrm{ml}$. The remainder of her differential blood counts and a co- agulation test were normal. An assay for antiplatelet antibodies (IgG and IgM) was positive. An oral corticosteroid treatment (prednisone, $0.5 \mathrm{mg} / \mathrm{kg}$, daily) was initiated. After 2 weeks of treatment, her platelet count increased to $89 \times 10^{3} / \mathrm{ml}$.

On February 8, 2006, after her platelet count had recovered in response to the oral corticosteroid treatment, a liver biopsy was performed to access the severity of the AILD. Marked lymphocyte infiltration and moderate fibrosis were observed in the portal area, but no other signs of an injured septal or interlobular bile duct were seen.

Thrombocytopenia occasionally occurs after routine immunization in children. Although serious autoimmune side effects are rare and most of them are transient, there are arguments against routine vaccination in patients with autoimmune diseases. On the other hand, Arakawa et al. [2] reported that ITP was rarely observed as a disease concomitant with AILD. This is apparently the first case report of a patient with AILD who developed ITP after receiving an influenza vaccine. UDCA was effective for this case. Moreover, primary biliary cirrhosis $(\mathrm{PBC})$ is an AILD characterized by the presence of AMA. The liver damage in this case may have been related to $\mathrm{PBC}$. $\mathrm{PBC}$ is known to be associated with several autoimmune

\section{KARGER}

() 2008 S. Karger AG, Basel

Fax +4161306 1234 E-Mail karger@karger.ch www.karger.com
0012-2823/08/0774-0159\$24.50/0

Accessible online at:

www.karger.com/dig
Satoshi Mamori, MD

Division of Gastroenterology and Hepatology

Department of Internal Medicine, The Jikei University School of Medicine

3-25-8 Nishi-shinbashi, Minato-ku, Tokyo 105-8461 (Japan)

Tel. +81 33433 1111, ext. 3201, Fax +81 33435 0569, E-Mail m8583jp@yahoo.co.jp 
diseases, including Hashimoto's thyroiditis. ITP is another autoimmune condition resulting in increased platelet destruction. Previous reports of patients with ITP developing into $\mathrm{PBC}$ have been published [2, 3]. Although some reports have described concomitant ITP in PBC patients not undergoing immunosuppressive therapies $[4,5]$, the present report describes an el- derly patient with PBC who developed ITP following an influenza vaccine. The pathogenesis of thrombocytopenia in patients with PBC is usually attributed to the splenic pooling of platelets. In addition, Panzer et al. [6] showed platelet-reactive antibodies to bind to the $70-\mathrm{kDa}$ mitochondrial antigen M2 in a patient with PBC. These findings suggest that immune-mediated platelet destruction may to some extent play a role in the pathogenesis of thrombocytopenia in PBC. The present report also supports the theory that the influenza vaccine can exacerbate potential immunemediated platelet destruction in AILD patients, thus leading to severe autoimmune side effects.

\section{References}

1 Geier MR, Geier DA, Zahalsky AC: Influenza vaccination and Guillain-Barré syndrome small star, filled. Clin Immunol 2003;107: 116-121.

2 Arakawa Y, Amaki S, Miyakawa H, et al: PBC-AIH overlap syndrome with concomitant ITP and Hashimoto's disease with positivity for anti-centromere antibody. J Gastroenterol 2004;39:490-495.
-3 Mizukami Y, Ohhira M, Matsumoto A, et al: Primary biliary cirrhosis associated with idiopathic thrombocytopenic purpura. J Gastroenterol 1996;31:284-288.

4 Fickert P, Trauner M, Sill H, Hinterleitner TA, Stauber RE: Successful steroid treatment of idiopathic thrombocytopenic purpura after orthotopic liver transplantation for primary biliary cirrhosis. Am J Gastroenterol 1998;93:1985-1986.

5 Yoshida EM, Mandl LA, Erb SR, Buckley AB Scudamore $\mathrm{CH}$, Buskard NA: Idiopathic thrombocytopenic purpura in a liver transplant recipient with previous primary biliary cirrhosis. J Clin Gastroenterol 1997;24:274275.
-6 Panzer S, Penner E, Nelson PJ, Prochazka E, Benda $\mathrm{H}$, Saurugger PN: Identification of the platelet glycoprotein IIb/IIIa complex as a target antigen in primary biliary cirrhosisassociated autoimmune thrombocytopenia. Evidence that platelet-reactive autoantibodies can also bind to the mitochondrial antigen M2. J Autoimmun 1990;3:473-483. 